cifics for the same; while a due insight into its complications, and a careful search after the precedents and tendencies thereof. would induce us to adopt rational means rather than be over-confident in any theory.

Debility would appear to me to be far less frequently a primary than a secondary condition. By the term primary debility, I would be understood to infer a cundition of simply depressed constitutional power, and by the term secondary debility to represent a state in which debility is the most characteristic symptom, and the one chiefly complained of by the sufferer, yet has been really induced by some perverted condition of the assimilative or nutritive functions, or the poison of some disease. In primary debility we have a condition predisposing to innu. merable diseases which may be generally averted, and the constitutional state corrected by stimulants, nutritious food and fresh air. In secondary debility we have invariably more than lost tone to restore; some blood-derangement to attend to, perverted functions to correct and complications to remove. Hence we oftentimes find that stimulants, when trusted to alone, prove irritants, in influencing a quicker circulation of the vital fluid through a system in a hyperæsthetic condition; while the blood in its depraved condition is incapable of affording a sufficiency of nutritive elements, or of sustaining the vital processes to a healthy standard.

\section{CASE OF RUPTURE OF THE AORTA WITHIN THE PERICARDIUM; WITH REMARKS.}

By Arthur Bracey, Esq., Surgeon to the Birmingham and Midland Eye Hospital.

THE subject of this accident was an unmarried woman, occupying a comfortable position as lady's maid, and 29 years of age.

She went in a cab, on the morning of April 4th, 1866, to see my father, as she was suffering from pain in her chest, and general uneasiness. She did not, however, meet with him, and returned home, where she had not long been, before she fell down in a kind of fit, at the same time giving a piercing shriek. I was then hastily called to her, and found her lying upon a couch, extremely pale, cold, and pulseless, but apparently sensible. After I had remained with her half an hour, she said she wished to go upstairs, and she walked up without much difficulty. She was placed in bed, and became warmer and less pale. In the evening she was more comfortable, but complained very much of pain in her chest, shoulders, and back of her neck; she did not refer any of her sensations to the immediate position of her heart.

At half-past three the following morning, fifteen hours after the first attack, I was again called to see her, and found her dead. I was told she had been, within three minutes of her death, quite calm, and talking rationally to her friends; there had been nothing noticed in her manner to indicate approaching dissolution. She had suddenly given three or four short and painful cries, thrown her arms wildly about, fallen backwards, and expired.

Secrio Cadaveris, thirty-six hours after death. On opening the chest, the pericardium was seen to be greatly distended, and, when divided, was found to contain a large quantity of bloody serum, and a firm clot which surrounded and embraced the heart. The amount of the contents was as nearly as possible one pint by measure.

On the external surface of the aorta, posteriorly, and about half an inch from its commencement, was a small and unevenly margined aperture, rather less than an eighth of an inch in diameter, communicating with the interior of the vessel. The areolar tissue in its neighbourhood was much infiltrated with blood, but there were no signs of any inflammatory products. There was no external wound of the heart. The interior of the aorta presented a remarkable appearance. Just above the semilunar valves was a rent extending through the internal and middle coats, completely following the circumference of the vessel in a spiral manner. The edges of the rent were widely separated, leaving only the external coat to complete the wall of the artery, and in this space was the aperture previously described.

A peculiarity existed in the semilunar valves themselves. One valve appeared complete, but the other two had no perfect division between them, and were thrown into one large fold; a rudimentary loop was present, but the arrangement was practically bivalvular instead of being trivalvular. The aorta was wider at its arch than normal, and its left margin formed a larger curve. My friend Mr. Furneaux Jordan has kindly examined a portion of the artery microscopically, and has found that the inner and middle coats contained a large number of small (young) nucleated cells; there were also elongated cells, with two or three nuclei, many of which were swollen, cloudy, and more or less opaque. The centres of cell-activity seemed very numerous and appeared to separate, or lie between, bands of fibrous and elastic tissue, in which the tissue-elements were only slightly, or not at all, changed. Fat-granules of various sizes were present in every part of the field.

The patient was a most abstemious person; she had spent an easy existence; but her health had never been very good, and, for some months, she had been in a desponding state of mind, so much so, that a fortnight previously to her death she attempted to commit suicide in rather a novel manner. She tied a tape tightly around her neck when she went to bed at night, allowing it to remain until the morning, when it was cut away by her friends. A very considerable effusion of blood under the conjunctivæ of both eyes, resulted from this compression.

The following remarks have suggested themselves to me. There was evidently an interval of time between the rupture of the inner arterial coats and the formation of the aperture in the outer coat. I am inclined to believe that the former took place in the morning at the time of the fainting fit; the accompanying shock to the system then prevented the stream of blood from passing with its usual force, and thus a further lesion was delayed. Fifteen hours after, however, when reaction was established, the areolar coat of the artery, which alone remained, yielded, blood escaped rapidly into the pericardial sac, and, exerting its pressure upon the walls of the heart, arrested its action, and death quickly ensued. It may be suggested that the earliest splitting of the inner coats took place at the time of the strangulation, but then I think there would have been some effusion of reparative material; most probably also a dissecting aneurism would have been formed, and perhaps considerably advanced.

The absence of any sudden mental emotion or violent bodily action makes a difficulty in determining the exact cause of the rupture, yet the microscopical examination has shown that which the unassisted eye could never have demonstrated; viz., that the vessel, though apparently diseased to a limited extent only, was nevertheless much altered in its minute structural arrangement, and liable at any time to such an accident as befel it. The pecu-
liar arrangement of the valves, too, must have thrown 
considerable strain on the left side of the vessel, and it was here probably that the rent commenced.

The specimen is preserved in the museum at Sydenham College, Birmingham.

\section{REMARKS ON SYPHILISATION.}

By Grorar Gaskorn, Esq., Surgeon, Chevalier of the Order of Christ, Portugal; Surgeon to the Artists' Benevolent Fund; formerly House-Surgeon and House-Pupil, St. George's Hospital.

$$
\text { [Continued from p. } 518 \text { of vol. i for 1866.] }
$$

Is our last we had to consider that argument of Henry Lee, in which he rests his point wholly on the character of induration in the chancre, which is better described by Ricord as characteristic and complementary, not as a feature essential to the course of infection. We are surprised that a man who makes pretensions to philosophy should fix on a single sign or character as bringing about results which occur commonly enough without its presence or precedence; and this is what Mr. Lee does when he makes this induration a "potential" as well as an "essential" sign of syphilitic infection. The temptation which physicians are under to mistake effects for causes, is favoured by our habit of clinical study, which directs itself exclusively to the manifestations of the disease, apart from the consideration of cause; the natural bias of our minds leading us ever to contemplate one or more prominent symptoms as governing the rest; especially when priority in order of sequence gives a plea for power. But what need we seek for more in the present case than the virus, as a complete and sufficient cause for all the phenomena? The thing itself is well enough understood by its effects. We need not be squeamish about Inaming it, like some pseudo-philosophers who would narrow the field of human thought till a man believed in nothing but the food between his teeth. We say fearlessly, then, that there is no stronger proof of the existence of a thing than the multiplied and long evidence we have of its action or of its being acted on; and in this we believe not to err against the laws of ancient or modern thought; and that is the case in the instance before us; and where the cause is one, its manifestations will vary according to the inconstant conditions of the matter subject to its operation.

As to this contagion of syphilis, we know of it by its persistency and generative power; and we cannot but recognise its modifications no less than its integrity. There is syphilitic matter which is like a searing iron in its action on the tissues; and sometimes we have ulceration and loss of tissue in its mildest forms; not seldom there is an excessive destruction and shedding of cell-formations which escape in the form of pus; and perhaps such celldeath is not, then, the single and exclusive effect of the virus on the economy. At other times, there is matter which enters the human frame with scarce a blot or stain of discoloration on the surface, yet it taints the blood and torments the body for years; and there is also that well recognised form, occurring chiefly in the male sex, which is capped by induration, such as is described by Mr. Lee. All these are primary manifestations; and the same may be said of those which are secondary-that induration, of whatever kind, is not a necessary complication in them.

But, if generally in the course and train of contagion like produces like-just as mumps from malaria produces mumps by contagion, and not an intermittent fever-we are not driven on that account to accept duality or a plurality of causes. We take into consideration that both the virus and its field of operation vary much; and especially do we hesitate when we see that the very men who dogmatise upon this subject, and offer us the conclusions of their narrow experience in a path confessedly difficult, shun and avoid a wider arena of experiment which is thrown open to them, and would fain bar and lock the doors. When a man has practised syphilisation, he may be allowed to have an opinion of his own on these questions, and may be excused from accepting theories which are no longer reconcileable with his everyday experience. And when Daniellsen, the Norwegian professor, says that he has seen thousands of artificial ulcers, and that (with one exception) they were all chancroids, we know that he means no more but that they were all of them non-indurated sores; and, being such, he concludes that they can in no way affect the system, or, at least, ought not to affect it. We understand that very well. "The long established axiom" of M. Clerc, that the chancroid does not infect, holds him fast; and he has bent his mind to these established axioms. In five lepers on whom he inoculated from the soft chancre, he did not get the result he thought himself entitled to expect, according to his interpretation of unity; viz., secondary syphilis ; and because he did not get these particular forms of disturbed function, therefore he falls in, it would seem, with the extreme opinion, that the direct operation of such chancres is exclusively local and limited to the spot on which they grow; if neither one, nor two, nor three, nor thirty-six, nor a hundred and thirty-six, induce secondary syphilis, then he feels justified in asserting that no number will do so. Failing the manifestations of secondary syphilis on the subjects of experiment, he negatives the possibility of all or any other influence upon the frame whatever as impossible, and in this he does not, as it would seem to us, betray the philosopher; but, in preferring the "long established axiom", he brings the professor into view.*

Much has been said of his sixth case, where, after 287 inoculations on a leper made with the secretion of soft sores, accidental inoculation from an indurated sore produced a hard chancre, after which constitutional syphilis ensued; showing that, at the time this happened, no immunity was acquired. Now, although immunity commonly follows at the end of 350 inoculations or so, yet the range is wide and uncertain; and in this case the patient had not reached that point of immunity, and what followed from inoculation of the hard chancre was a matter of course.

Boeck, on the other hand, syphilised a woman who had obstinate chronic eczema; and, even as late as five years after, on repeated trials, she could not be inoculated from the hard chancre, nor were there any secondaries in her case. Since that time, or thereabouts, Boeck has inoculated only with the secretion of the hard chancre and its product in direct linear series. This is a saying hard to be borne by those who blindly follow the dictamina of Messrs. Ricord, Fournier, and Rollet; and then it becomes a question what liberty we are allowed in departing from their ideas in England and in Norway, and what amount of "initiative" is forbidden in Europe by these "French swells", who think all linen dirty that is not washed in French waters.

In producing immunity, it is necessary not to stop

* We are far from judging that this criticism applies with correctness to the opinions of M. Daniellsen; it is only meant for the interpretation or misinterpretation of them given in our manuals. (See Bumstead On Syphilis.) 